\title{
Analytical GMD formulas for mutual inductance calculation of multilevel interconnects
}

\author{
Hideki Shima $^{1 \mathrm{a})}$, Toshimasa Matsuoka ${ }^{1}$, and Kenji Taniguchi ${ }^{1}$ \\ ${ }^{1}$ Department of Electronics and Information Systems, Osaka University \\ 2-1 Yamada-oka, Suita-shi, Osaka 565-0871, Japan \\ * Presently, Texas Instruments Japan Ltd. \\ 1-8-30 Tenma-bashi, Kita-ku, Osaka-shi, Osaka 530-6026, Japan. \\ a)h-shima@ti.com
}

\begin{abstract}
A set of new analytical formulas is proposed for determining the geometric mean distance (GMD) for use in the Greenhouse method to calculate the inductances of three-dimensional inductors composed of on-chip interconnects. The expressions are derived using a graphical method based on the integral form of the GMD equation. The mutual inductances of coupled interconnects are computed for given layout geometries using the proposed formulas, even when the conductors are partially overlapped in different metal layers. The inductances computed using the formulas agreed well with those generated by an accurate commercial field solver, differing by less than $1 \%$ across a variety of the conductor geometries.
\end{abstract}

Keywords: Greenhouse method, geometric mean distance, on-chip interconnect, three-dimensional inductor, mutual inductance, closedform expression

Classification: Integrated circuits

\section{References}

[1] H. Y. D. Yang, "Design considerations of differential inductors in CMOS technology for RFIC," IEEE RFIC Symp. Dig., TX, pp. 449-452, June 2004.

[2] A. Zolfaghari, A. Chan, and B. Razavi, "Stacked inductors and transformers in CMOS technology," IEEE J. Solid-State Circuits, vol. 36, no. 4, pp. 620-628, April 2001.

[3] P. Park, C. S. Kim, M. Y. Park, S. D. Kim, and H. K. Yu, "Variable inductance multilayer inductor with MOSFET switch control," IEEE Electron Device Lett., vol. 25, no. 3, pp. 144-146, March 2004.

[4] H. M. Greenhouse, "Design of planar rectangular microelectronic inductors," IEEE Trans. Parts. Hyb. Packag., vol. 10, no. 2, pp. 101-109, June 1974.

[5] F. W. Grover, "The calculation of the inductance of single-layer coils and spirals wound with wire of large cross section," Proc. the Institute of Radio Engineers, vol. 17, no. 11, pp. 2053-2063, Nov. 1929. 
[6] F. W. Grover, Inductance Calculations, Van Nostrand, New York, 1946.

[7] T. Yamamoto, M. Ikeda, and K. Asada, "Inductance calculation for rectangle section form wiring using GMD," Proc. IEICE Gen. Conf., A-3-16, p. 83, March 2003.

[8] H.-M. Hsu, "Analytical formula for inductance of metal of various widths in spiral inductors," IEEE Trans. Electron Devices, vol. 51, no. 8, pp. 1343-1346, Aug. 2004.

[9] Q3D Extractor, Ansoft Corp., Pittsburgh, PA.

[10] Raphael, Synopsys, Inc., Mountain View, CA.

[11] M. Kamon, M. J. Tsuk, and J. White, "FASTHENRY: A multipoleaccelerated 3-D inductance extraction program," IEEE Trans. Microwave Theory Tech., vol. 42, no. 9, pp. 1750-1758, Sept. 1994.

[12] A. Kurokawa, K. Hachiya, T. Sato, K. Tokumasu, and H. Masuda, "Fast on-chip inductance extraction of VLSI including angled interconnects," IEICE Trans. Fundamentals, vol. E86-A, no. 4, pp. 841-845, April 2003.

\section{Introduction}

Continued improvements in semiconductor process technology have enabled the design of advanced on-chip inductors [1]-[3] using multilevel interconnects. Greenhouse [4] reported a technique based on analytical expressions to calculate the inductance of planar rectangular spiral inductors. Accurate results can be obtained with this algorithm, but the expression for geometric mean distance (GMD) can only be applied to aligned conductors in the same layer that have the same width and negligible thickness. The precomputed tables reported in Refs. [5] and [6] can be employed to calculate the GMD of two rectangular cross sections with finite thickness. The interconnection layout, however, is restricted to aligned cases. Expressions for aligned conductors of given widths and thicknesses were reported in Refs. [7] and [8], but expressions for interconnects placed in different metal layers were not addressed. Although three-dimensional (3D) field solvers [9]-[11] can accurately calculate inductances of multilevel conductors, they are timeconsuming compared to the analytical technique due to the mesh generation algorithm [7, 12].

This paper presents a set of simple and accurate formulas for calculating the GMD of multilevel on-chip interconnects to be used in the Greenhouse method. The technique to derive the proposed expressions is based on the integral form of the GMD equation. The formulas include the geometric parameters of the metal lines, allowing for the independent scaling of conductor widths and thicknesses. The practical application of the formulas in the Greenhouse method is demonstrated with the results from commercial field solver Ansoft Q3D Extractor [9].

\section{Geometric Mean Distance Expressions}

The proposed set of GMD formulas for interconnects in different metal layers (see Figs. 1 (a)-(e)) was derived as a function of the GMD formula for aligned 


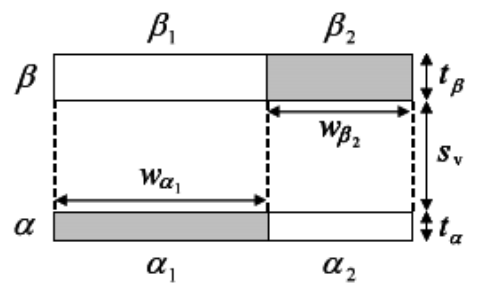

(a)

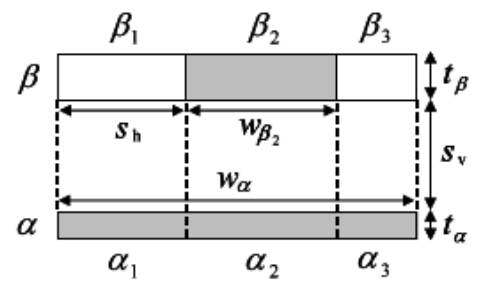

(c)

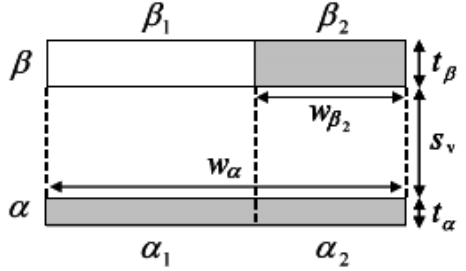

(b)

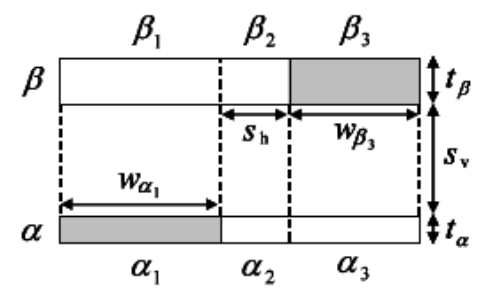

(e)

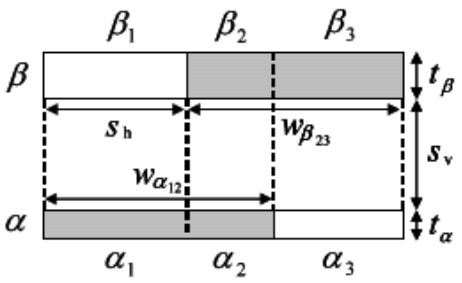

(d)

Fig. 1. Cross sectional views of multilevel conductors used in VLSI circuits. The shaded rectangles depict the cross sections of the interconnects while the others denote the segments used to derive the GMD of the coupled interconnects. The geometric parameters of the conductors, the width $w$, the vertical and horizontal spacings $s_{\mathrm{v}}$ and $s_{\mathrm{h}}$, and the thickness $t$, are shown in the figures. The subscripts on the widths and thicknesses indicate the corresponding rectangles.

conductors $[7,8]$. The integral form of the GMD equation used to derive the formulas is given by

$$
\log R_{i j}=\frac{1}{S_{i} S_{j}} \iint \log r d S_{i} d S_{j} \quad(i \neq j)
$$

where $R_{i j}$ is the GMD of rectangular cross sections $i$ and $j, r$ is the distance between every pair of points in the sections $i$ and $j$, and $S_{i}$ and $S_{j}$ are the areas of the respective sections.

The concept for the derivation is explained using the example in Fig. 1 (a). Aligned stacked rectangles $\alpha$ and $\beta$ are decomposed into aligned stacked segments including the two conductors $\alpha_{1}$ and $\beta_{2}$ of rectangular cross section. It should be noted that the GMD for case (a) is derived from Eq. (1) when the unknown term, the double integral of $\log r$ over the pair of rectangular cross sections $(i, j)$, is obtained for the pair of rectangles $(i, j)=\left(\alpha_{1}, \beta_{2}\right)$. This decomposition yields the relationship between the double integral of $\log r$ over the pair of rectangles $(i, j)=\left(\alpha_{1}, \beta_{2}\right)$ and those of aligned rect- 
(a)

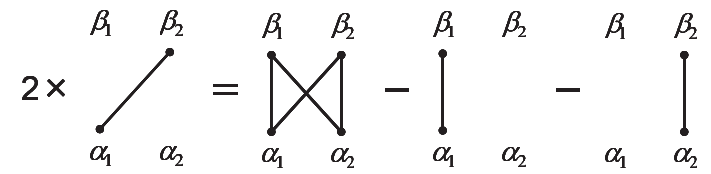

(b)

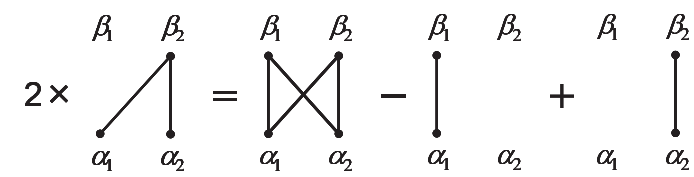

(c)

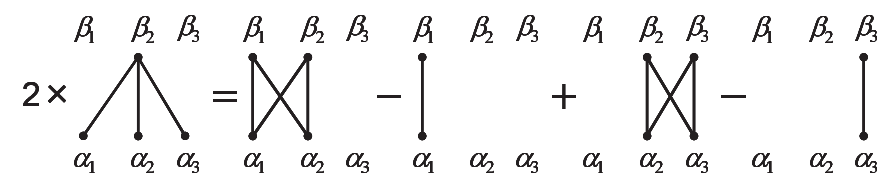

(d)

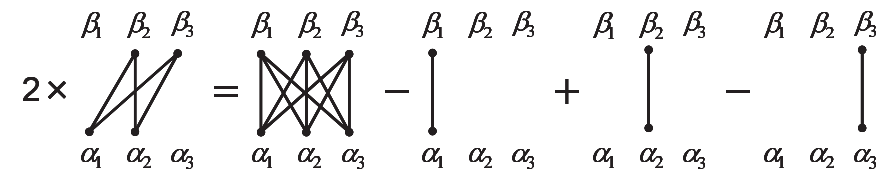

(e)

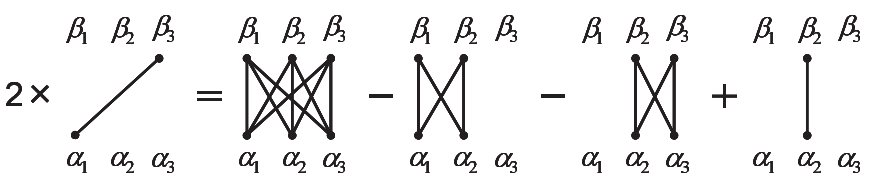

Fig. 2. Graphical expressions for deriving proposed GMD formulas in cases (a)-(e) in Fig. 1. The terms in the expressions represent the double integrals in Eq. (1) over the pairs of segments denoted by the solid lines connecting the segments between aligned rectangles $\alpha$ and $\beta$. The details are described in the text.

angles $(i, j)=(\alpha, \beta),\left(\alpha_{1}, \beta_{1}\right)$ and $\left(\alpha_{2}, \beta_{2}\right)$ as the graphical expression shown in Fig. 2(a). The terms in the graphical expression represent double integrals over the pairs of rectangles indicated by solid lines connecting the segments. For instance, the term in the left hand side of the graphical expression for case (a) denotes twice the double integral of $\log r$ over the segments $(i, j)=\left(\alpha_{1}, \beta_{2}\right)$. It should be noted that the integral value over the pair of cross coupled segments $(i, j)=\left(\alpha_{2}, \beta_{1}\right)$ is equal to that of the cross coupled segments $(i, j)=\left(\alpha_{1}, \beta_{2}\right)$ even if the segments have different thicknesses, as in Fig. 1 (a). The integration regions indicated by the each term in the right hand side of the expression are composed of coupled aligned rectangles to obtain the GMDs of aligned rectangles. This graphical description facilitates the derivation of the GMD formula because the relationship between double integrals over cross coupled and aligned rectangles can be found easily by performing arithmetic operations directly on the corresponding solid lines between the terms shown in the figure. The graphical expression for case (a) can be reduced to the following mathematical scheme:

$$
2 F_{\alpha_{1} \beta_{2}}=F_{\alpha \beta}-F_{\alpha_{1} \beta_{1}}-F_{\alpha_{2} \beta_{2}}
$$


where

$$
F_{i j} \equiv \iint \log r d S_{i} d S_{j} \quad(i \neq j)
$$

where $i$ and $j$ denote the regions of the rectangles to be integrated. Using Eqs. (1), (2) and (3), the resulting formula for case (a) is given by

$$
\begin{aligned}
\log R_{\alpha_{1} \beta_{2}}^{\mathrm{a}}= & \frac{1}{2 S_{\alpha_{1}} S_{\beta_{2}}}\left(S_{\alpha} S_{\beta} \log R_{\alpha \beta}-S_{\alpha_{1}} S_{\beta_{1}} \log R_{\alpha_{1} \beta_{1}}\right. \\
& \left.-S_{\alpha_{2}} S_{\beta_{2}} \log R_{\alpha_{2} \beta_{2}}\right)
\end{aligned}
$$

where $R_{\alpha_{1} \beta_{2}}^{\mathrm{a}}$ is the GMD for case (a). $R_{\alpha \beta}, R_{\alpha_{1} \beta_{1}}$ and $R_{\alpha_{2} \beta_{2}}$ are the GMDs for aligned stacked rectangles $(i, j)=(\alpha, \beta),\left(\alpha_{1}, \beta_{1}\right)$ and $\left(\alpha_{2}, \beta_{2}\right)$, respectively.

The GMD formulas for cases (b)-(e) are derived using the graphical method as described in case (a). Figures 2 (b)-(e) show the graphical expressions derived by performing arithmetic operations on the solid lines. The resulting formulas are given by

$$
\begin{aligned}
\log R_{\alpha \beta_{2}}^{\mathrm{b}}= & \frac{1}{2 S_{\alpha} S_{\beta_{2}}}\left(S_{\alpha} S_{\beta} \log R_{\alpha \beta}-S_{\alpha_{1}} S_{\beta_{1}} \log R_{\alpha_{1} \beta_{1}}\right. \\
& \left.+S_{\alpha_{2}} S_{\beta_{2}} \log R_{\alpha_{2} \beta_{2}}\right) \\
\log R_{\alpha \beta_{2}}^{\mathrm{c}}= & \frac{1}{2 S_{\alpha} S_{\beta_{2}}}\left(S_{\alpha_{12}} S_{\beta_{12}} \log R_{\alpha_{12} \beta_{12}}-S_{\alpha_{1}} S_{\beta_{1}} \log R_{\alpha_{1} \beta_{1}}\right. \\
& \left.\quad+S_{\alpha_{23}} S_{\beta_{23}} \log R_{\alpha_{23} \beta_{23}}-S_{\alpha_{3}} S_{\beta_{3}} \log R_{\alpha_{3} \beta_{3}}\right) \\
\log R_{\alpha_{12} \beta_{23}}^{\mathrm{d}}= & \frac{1}{2 S_{\alpha_{12}} S_{\beta_{23}}}\left(S_{\alpha} S_{\beta} \log R_{\alpha \beta}-S_{\alpha_{1}} S_{\beta_{1}} \log R_{\alpha_{1} \beta_{1}}\right. \\
& \left.\quad+S_{\alpha_{2}} S_{\beta_{2}} \log R_{\alpha_{2} \beta_{2}}-S_{\alpha_{3}} S_{\beta_{3}} \log R_{\alpha_{3} \beta_{3}}\right) \\
\log R_{\alpha_{1} \beta_{3}}^{\mathrm{e}}= & \frac{1}{S_{\alpha_{1}} S_{\beta_{3}}}\left(S_{\alpha} S_{\beta} \log R_{\alpha \beta}-S_{\alpha_{12}} S_{\beta_{12}} \log R_{\alpha_{12} \beta_{12}}\right. \\
& \left.-S_{\alpha_{23}} S_{\beta_{23}} \log R_{\alpha_{23} \beta_{23}}+S_{\alpha_{2}} S_{\beta_{2}} \log R_{\alpha_{2} \beta_{2}}\right)
\end{aligned}
$$

where $R_{\alpha \beta_{2}}^{\mathrm{b}}, R_{\alpha \beta_{2}}^{\mathrm{c}}, R_{\alpha_{12} \beta_{23}}^{\mathrm{d}}$ and $R_{\alpha_{1} \beta_{3}}^{\mathrm{e}}$ are the GMDs for the respective cases (b)-(e). $\alpha_{12}, \alpha_{23}, \beta_{12}$ and $\beta_{23}$ represent rectangles $\alpha_{1} \cup \alpha_{2}, \alpha_{2} \cup \alpha_{3}, \beta_{1} \cup \beta_{2}$, and $\beta_{2} \cup \beta_{3}$, respectively. Note that Eqs. (4)-(8) are determined from the layout geometries of the coupled conductors in Fig. 1 because the GMD for aligned conductors can be computed from a known analytical expressions that include the geometric parameters $[7,8]$.

\section{Verification}

Figure 3 shows the mutual inductances for cases (a)-(e) calculated using the analytical expression in the Greenhouse method [4] in conjunction with the proposed formulas. The mutual inductances were simulated using Ansoft Q3D Extractor [9]. Note that the GMD for aligned conductors used in the formulas were determined from the expression in Ref. [7] that uses the GMD of a rectangle from itself with geometric parameters $[5,6]$. The vertical spacing $s_{\mathrm{V}}$ on the $x$-axis and the widths $w_{\beta_{2}}, w_{\beta_{23}}$ and $w_{\beta_{3}}$ of each configuration (see Fig. 1) are varied. The results from the proposed expressions agree well with those of the field solver, differing by less than $1 \%$ even for the 

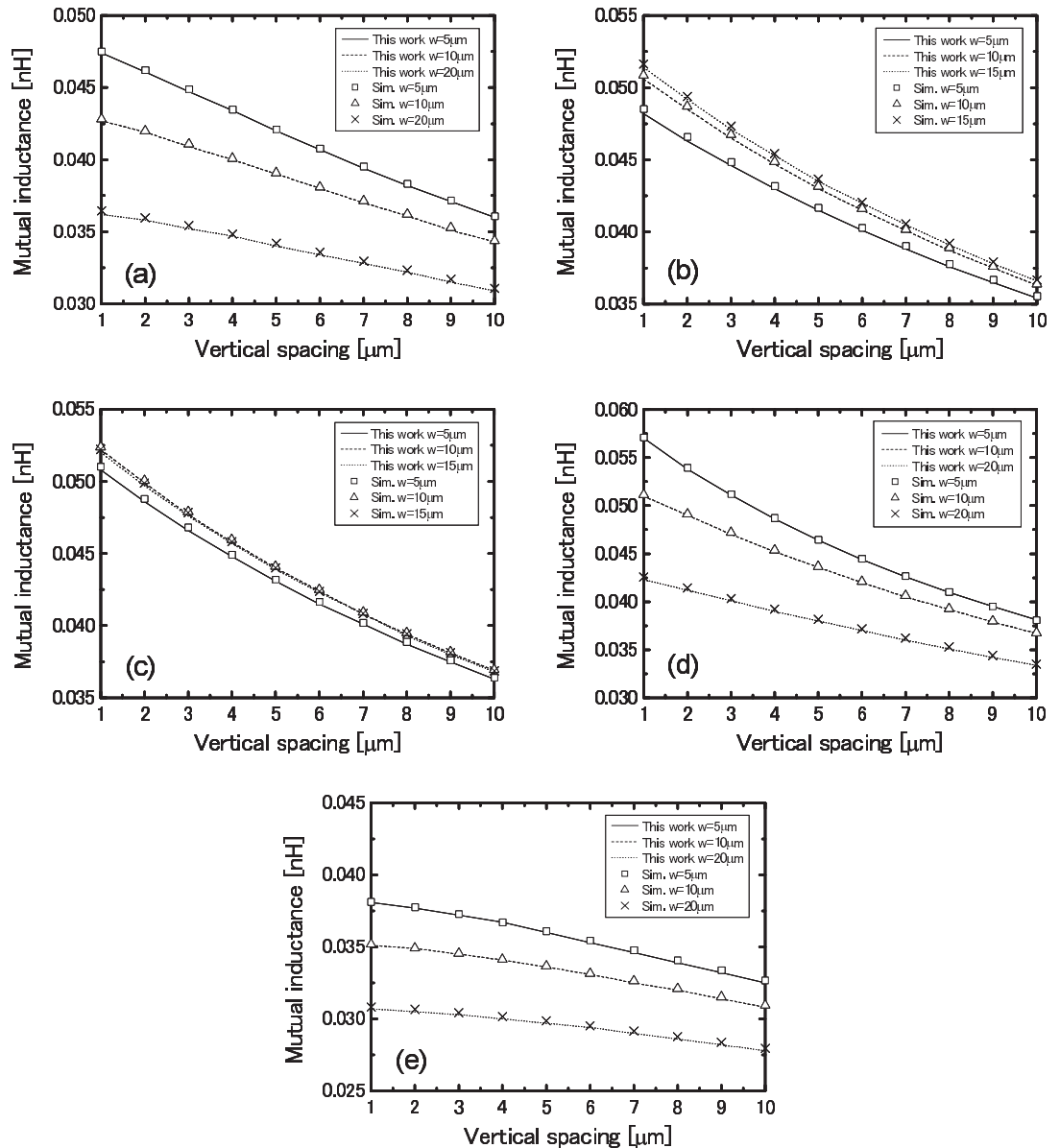

Fig. 3. Mutual inductances calculated using the proposed formulas and a field solver for cases (a)-(e). A length of $100 \mu \mathrm{m}$ is used in all cases, together with the following in the individual cases: (a) $w_{\alpha_{1}}=$ $10 \mu \mathrm{m}, w_{\beta_{2}}=w, t_{\alpha}=1 \mu \mathrm{m}$ and $t_{\beta}=2 \mu \mathrm{m}$ (b) $w_{\alpha}=20 \mu \mathrm{m}, w_{\beta_{2}}=w, t_{\alpha}=1 \mu \mathrm{m}$ and $t_{\beta}=2 \mu \mathrm{m}$ (c) $w_{\alpha}=20 \mu \mathrm{m}, w_{\beta_{2}}=w, s_{\mathrm{h}}=2 \mu \mathrm{m}, t_{\alpha}=1 \mu \mathrm{m}$ and $t_{\beta}=2 \mu \mathrm{m}(\mathrm{d}) w_{\alpha_{12}}=10 \mu \mathrm{m}, w_{\beta_{23}}=w, s_{\mathrm{h}}=$ $6 \mu \mathrm{m}, t_{\alpha}=1 \mu \mathrm{m}$ and $t_{\beta}=2 \mu \mathrm{m}$ (e) $w_{\alpha_{1}}=10 \mu \mathrm{m}$, $w_{\beta_{3}}=w, s_{\mathrm{h}}=5 \mu \mathrm{m}, t_{\alpha}=1 \mu \mathrm{m}$ and $t_{\beta}=2 \mu \mathrm{m}$.

narrow spacing case. The inductance decreases with increasing conductor spacing because the magnetic coupling between the conductors is inversely proportional to the spacing of the metal lines. These plots indicate that the Greenhouse method can be extended to multilevel interconnects to a high degree of accuracy by applying the proposed formulas.

\section{Conclusion}

Accurate analytical formulas for obtaining the GMD of multilevel conductors for the Greenhouse method have been presented. The integral form of the GMD equation was used to derive the GMD expressions for multilevel metal lines. The mutual inductances of multilevel conductors calculated using the proposed formulas matched those derived with a commercial field 
solver within $1 \%$. The proposed expressions are fully scalable and computationally efficient for calculating mutual inductances of interconnects for 3D inductors in VLSI circuits. 